\title{
Lifetime visual prognosis for patients with primary open-angle glaucoma
}

Department of

Ophthalmology, Norfolk and Norwich University Hospital, Norwich, UK

Correspondence: T Eke, Department of Ophthalmology, Norfolk and Norwich University Hospital, Colney Lane, Norwich NR4 7UZ, UK Tel: +44 1603288 578; Fax: + 441603288261 E-mail: tom.eke@ nnuh.nhs.uk

Received: 31 October 2004 Accepted in revised form: 5 January 2006

Published online:

17 February 2006

Proprietary interests/ research funding: Nil

Presentations at Meetings: Poster Oxford

Ophthalmological Congress July 2004

\begin{abstract}
Aim To investigate final visual outcome in primary open angle glaucoma (POAG) including low-tension glaucoma (LTG). Methods Retrospective review of case notes for patients who died between 1999 and 2002. All were booked for a follow-up appointment in glaucoma clinic at time of death.
\end{abstract}

Results A total of 121 case notes were reviewed. In all, 113 patients had POAG and eight had LTG. All were White Caucasians. Mean ages at presentation and death were 74.6 (SD 9.6, range 49-94) and 81.9 (SD 8.3, range 51-98) years, respectively. Mean follow-up duration was 7.4 (SD 6.8, range up to 29) years. Average number of clinic visits was 18 (SD 17, range 1-95). At final visit, 50.4\% had cataract operations, and $45.5 \%$ had glaucoma operations. At final visit, vision was inadequate for driving in the UK in $47.1 \%$. In $18.2 \%$, this was due to glaucoma alone, while in $\mathbf{2 8 . 9 \%}$, other ocular pathologies contributed to poor vision. In all, $14 \%$ were eligible for partial sight certification, with $6.6 \%$ due to glaucoma alone. A total of $3.3 \%$ were eligible for blind certification, none due to glaucoma alone.

Conclusion This study shows that POAG does affect the quality of life, with regards to glaucoma clinic visits, eye drops, and surgical procedures. Most patients with treated POAG in Norfolk will retain useful vision for their whole life. A significant proportion of patients with POAG do lose vision resulting in driving ineligibility and certification as visually impaired, although actual blindness is uncommon.

Eye (2007) 21, 604-608. doi:10.1038/sj.eye.6702284; published online 17 February 2006

Keywords: glaucoma; lifetime outcome; blindness; partial sight; driving eligibility
GS Ang and T Eke

\section{Introduction}

Primary open-angle glaucoma (POAG) is the most common form of glaucoma. The estimated prevalence among adult Europeans is $2.4 \% .^{1}$ If untreated, visual defects may progress over many years and this can lead to blindness. A previous study of treated POAG patients in the United States found a 9\% probability of bilateral blindness and $26 \%$ probability of unilateral blindness at 20 years. ${ }^{2}$ In developing countries, POAG is a major cause of blindness, mainly due to poor access to screening and treatment. ${ }^{3-7}$ It is estimated that glaucoma is the second leading cause of visual loss worldwide, and is responsible for up to $17 \%$ of blind and partial sight registration in the United Kingdom. ${ }^{1,8-11}$

A newly diagnosed POAG patient will have concerns about the future. Will their eyesight remain good enough to retain their driving licence? Will they go blind completely? Despite the large amount of literature regarding glaucoma in the United Kingdom, there is little evidence to answer these questions. We attempted to answer this by reviewing the case notes of glaucoma patients who have recently died.

\section{Materials and methods}

This is a retrospective review of case notes. We looked at the case notes of glaucoma patients who died between 1999 and 2002. These patients had attended the glaucoma clinic at our Ophthalmology Department in Norwich, United Kingdom, and were booked for a followup appointment in the glaucoma clinic at time of death. We included patients who had become blind prior to death as long as they were still under glaucoma follow-up. Patients were excluded if they were lost to follow-up or discharged, rather than deceased. We analysed 
data for patients with a clinical diagnosis of POAG or low-tension glaucoma (LTG), and excluded patients with ocular hypertension and other glaucoma diagnoses.

End point was defined as the last glaucoma clinic visit before death. Data collected for each patient included the clinical diagnosis, age at presentation, age at death, driving eligibility, partial sight or blind certification eligibility, number of glaucoma clinic visits, number of surgical and laser procedures, duration of follow-up, and concurrent eye pathology. We reviewed the notes to ensure that we agreed with the glaucoma diagnosis that had been made by the clinicans who treated each patient. In assessing the number of clinic visits, we only counted visits to the glaucoma clinic and excluded preoperative assessments, admissions, first day postoperative checks, and other clinic visits, such as for macular disease. It was not possible to collect data on the causes of death in all patients.

Driving eligibility of patients was based on current United Kingdom Driver and Vehicle Licensing Agency (DVLA) guidelines (www.dvla.gov.uk/at_a_glance/ ch6_visual.htm (accessed July 2004)). For central visual acuity, this is expressed in terms of reading a car number plate. We used the Snellen acuity of 6/12 or worse to indicate a DVLA 'fail' in terms of visual acuity. For visual fields, the DVLA requires no 'significant' loss within the central $20^{\circ}$, or within a $120^{\circ}$ zone along the horizontal meridian using the Esterman protocol. Most of our patients had not had Esterman visual fields performed, so visual fields, which were mainly monocular threshold fields, were assessed by one of us (TE) to estimate whether the binocular field would pass DVLA criterion. This was performed by merging the sensitivities at each visual field location of both eyes, based on the technique described by Crabb et al. ${ }^{12}$

Partial sight or blind registration eligibility of patients was based on visual acuity and field guidelines as stated on the United Kingdom BD8 (1990) certification form (www.dh.gov.uk/assetRoot/04/07/48/48/ 04074848.doc (accessed October 2005)). We considered a patient to be eligible for partial sight certification if the best-corrected visual acuity is worse than $6 / 60$, or $6 / 18$ with a gross visual field defect. The patient is eligible for blind certification if the best-corrected visual acuity is worse than $3 / 60$, or $6 / 60$ with a very constricted visual field especially if the inferior field is affected.

\section{Results}

Case notes were available for 173 patients who had died while awaiting review in glaucoma clinic from 1999 to 2002. Of these, 113 patients $(65.3 \%)$ had a clinical diagnosis of POAG, and eight patients $(4.6 \%)$ had LTG.
Further 52 patients were excluded from analysis because they had other diagnoses: 15 patients $(8.7 \%)$ had ocular hypertension, 11 patients $(6.4 \%)$ were glaucoma suspects, and 26 patients $(15.0 \%)$ had other forms of glaucoma including pigment dispersion, pseudoexfoliation, and narrow angle glaucoma. Results are presented for the patients with POAG and LTG only.

In the POAG and LTG group, all patients were white Caucasians. The mean age at presentation was 74.6 years (SD 9.6, range 49-94). The mean age at final glaucoma clinic visit was 81.9 years (SD 8.3, range 51-98). The mean follow-up duration was 7.4 years (median 4, SD 6.8, range up to 29 years). The average number of clinic visits was 18 (median 11, SD 17, range 1-95 visits). At presentation, the mean intraocular pressure was $25.6 \mathrm{mmHg}$ (median 24, SD 7.2, range 12-56). At last clinic visit, the mean intraocular pressure was $15.6 \mathrm{mmHg}$ (median 15, SD 4.3, range 2-32).

A total of 73 patients $(60.3 \%)$ underwent surgical interventions (cataract or glaucoma surgery or both). Table 1 summarises the ocular operations and lasers that these patients had. In all, 61 patients $(50.4 \%)$ had cataract operations, 55 patients $(45.5 \%)$ had glaucoma operations, and 16 patients (13.3\%) had a laser procedure. In all, 41 patients (33.9\%) had both glaucoma and cataract surgery. A total of 97 patients $(80.2 \%)$ were using topical glaucoma medication at last clinic visit.

Table 2 summarises the eligibility for driving for these patients. At presentation, 38 patients $(31.4 \%)$ had visual function that appeared to be inadequate for driving

Table 1 Surgical and laser procedures performed during the lifetime of 121 patients with POAG or LTG

\begin{tabular}{llc}
\hline Procedure & No. of eyes & $\begin{array}{c}\text { No. of POAG/LTG } \\
\text { patients }(\%)\end{array}$ \\
\hline Cataract surgery & $\begin{array}{l}\text { One eye } \\
\text { Two eyes }\end{array}$ & $27(22.3)$ \\
Trabeculectomy & $\begin{array}{l}\text { One eye } \\
\text { Two eyes }\end{array}$ & $29(24.1)$ \\
& One eye & $26(21.5)$ \\
Bleb needling & Two eyes & $3(2.5)$ \\
Redo trabaeculectomy & One eye & 0 \\
Tube procedure & Two eyes & $9(7.4)$ \\
& One eye & 0 \\
Laser trabaeculoplasty & Two eyes & 0 \\
& One eye & 0 \\
Cyclodiode & Two eyes & $4(3.3)$ \\
Ciliary ablation & Twe eye & $2(1.7)$ \\
\hline
\end{tabular}


Table 2 Driving eligibility for 121 patients with POAG or LTG

\begin{tabular}{llc}
\hline Time point for driving ineligibility & Reason for ineligibility & No. of POAG /LTG patients ineligible to drive (\%) \\
\hline At presentation & Glaucoma damage alone & $10(8.3)$ \\
& Glaucoma plus other pathology & $28(23.1)$ \\
& Total & $38(31.4)$ \\
At final clinic visit & Glaucoma damage alone & $22(18.2)$ \\
& Glaucoma plus other pathology & $36(28.9)$ \\
\hline
\end{tabular}

Comparison of eligibility for driving at presentation and at final visit before death. Eligibility for driving was estimated according to DVLA criteria (see text).

Table 3 Eligibility for partial sight or blind certification for 121 patients with POAG or LTG

\begin{tabular}{llcr}
\hline Time point for partial sight/blind eligibility & Reason for poor vision & No. of POAG/LTG patients (\%) \\
\cline { 2 - 3 } & & Partial sight eligible & Blind eligible \\
\hline At presentation & Glaucoma damage alone & $1(0.8)$ & 0 \\
& Glaucoma plus other pathology & $7(5.8)$ & 0 \\
& Total & $8(6.6)$ & 0 \\
At final clinic visit & Glaucoma damage alone & $8(6.6)$ & $9(7.4)$ \\
& Glaucoma plus other pathology & $17(14.0)$ & $4(3.3)$ \\
\hline
\end{tabular}

Comparison of visual function at presentation and at final visit before death.

standards; in 10 patients $(8.3 \%)$, this was due to glaucoma alone. At last visit, 57 patients (47.1\%) had visual function that appeared to be inadequate for driving standards; in 22 patients (18.2\%), this was due to glaucoma alone. The other causes of inadequate visual function were mainly cataract and macular degeneration. For those who eventually became ineligible to drive, mean time from presentation to ineligibility was 8.7 years (SD 6.7, range up to 27 years). The reasons for failing the DVLA driving criteria were inadequate visual acuity in 25 patients $(20.7 \%)$, and inadequate visual field in 32 patients $(26.4 \%)$.

Table 3 summarises the eligibility for blind or partial sight certification. No patient lost all perception of light. In all, 21 patients (17.3\%) were eligible for certification as blind or partially sighted at last visit. No patient was certified blind due to glaucoma damage alone; while eight patients $(6.6 \%)$ became eligible for partial sight certification from glaucoma alone. When other pathology, mainly macular degeneration, was taken into account, four patients (3.3\%) were blind eligible, and a total of 17 patients $(14.0 \%)$ were partial sight eligible at last clinic visit. At presentation to the glaucoma clinic, eight patients $(6.6 \%)$ were already eligible for partial sight certification. Of the remaining 13 patients $(10.7 \%)$, the mean duration to onset of eligibility for BD8 certification was 10.5 years from presentation (SD 8.1, range up to 27 years).

\section{Discussion}

This study estimates the lifetime prognosis for glaucoma patients, in terms of follow-up duration, number of clinic visits, and the number of laser and surgical procedures; and will therefore be of interest to patients, clinicians, and managers. Owing to the retrospective design of the study, there may be a possibility that the most elderly and infirm POAG patients may have been inadvertently excluded from the study as they were unable to attend the clinic appointment. Additionally, some patients may have been discharged because they had lost all vision, or returned to the care of their optometrist if the glaucoma was thought to be stable. This is a potential source of bias and may make our overall outcomes appear better than they really are. Unfortunately, we have no way of obtaining this information. A lifelong observational cohort study would be better placed to address the question of lifetime visual prognosis for POAG, but that would be extremely time consuming and costly.

It may be that these results are already 'out of date' due to changes in glaucoma management in recent years, 
including better screening and changes in the threshold for glaucoma diagnosis and treatment. We would therefore expect our current cohort of POAG patients to have a better prognosis. Studies showing that blindness from glaucoma occurring at an increasingly later age attribute this to an improvement in glaucoma treatment methods. ${ }^{8,13}$ It has been estimated that the mean time from initial visual field defect to death was 12.8 years in white European patients, suggesting that in the majority of treated glaucoma patients, the rate of progression of visual field loss should not lead to blindness in their lifetime. ${ }^{14,15}$ Our results confirm that the majority of treated POAG patients in Norfolk will not go blind before death. However, as our results were derived from a modest sample size of white Caucasians, there is uncertainty as to whether our conclusions can be applied as a whole to the larger and multiethnic population of patients with glaucoma in the United Kingdom.

For the DVLA to assess fitness to drive, current guidelines recommend using binocular Esterman visual field assessments using a target equivalent to the white Goldmann III4e settings are used. As only $3.4 \%$ of our patients had an Esterman plot in their case notes, we had to make an estimate of DVLA visual field eligibility based on monocular fields for most of our patients. ${ }^{16}$ Therefore, we made our estimate based on the method of merging sensitivity values at each visual field location from both eyes, as described by Crabb et al. ${ }^{12,16}$ We acknowledge that our method is unlikely to give a totally accurate estimate of DVLA eligibility in our patients, especially since it only considers the central visual field.

However, we felt that it should be included in this report because DVLA eligibility is important to most of our patients, and it is an end point that is easily understood by patients.

Hopefully, our current cohort of glaucoma patients will have even better results due to improvements in glaucoma screening (earlier diagnosis) and in glaucoma management. However, patients are also living longer, so that on average glaucoma patients will have the condition for longer, and this may impact negatively on the final visual outcomes. We will therefore be repeating this study in a few years' time.

We were pleased to see that the majority of our glaucoma patients maintain useful vision. However, a significant proportion does lose vision due to conditions other than glaucoma. If undetected and untreated, POAG does result in significant visual morbidity. Populationbased studies in countries with poor access to eye care have confirmed that untreated POAG is a major cause of blindness. $^{3-7}$ Our study shows that in Norfolk at least, and perhaps the rest of the United Kingdom, the great majority of white patients with diagnosed and treated
POAG will maintain some useful vision for the whole of their lives.

\section{Conclusion}

This study shows that a diagnosis of POAG does impact on the patient in terms of the duration and number of glaucoma clinic visits, and surgical procedures performed. However, the study will be reassuring to patients, because it shows that treated POAG is associated with a low lifetime risk of serious visual impairment. The majority of patients maintain useful vision through their lifetime, and actual blindness is uncommon. However, it also shows that a significant proportion loses vision resulting in driving ineligibility or partial sight certification. With improvements in glaucoma screening and management, it is hoped that our current cohort of glaucoma patients will have even better lifetime visual outcomes.

\section{Competing interests: Nil.}

\section{References}

1 Quigley HA. Number of people with glaucoma worldwide. Br J Ophthalmol 1996; 80: 389-393.

2 Hattenhauer MG, Johnson DH, Ing HH, Herman DC, Hodge DO, Yawn BP et al. The probability of blindness from open-angle glaucoma. Ophthalmology 1998; 105: 2099-2104.

3 Bourne RR, Sukudom P, Foster PJ, Tantisevi V, Jitapunkul S, Lee PS et al. Prevalence of glaucoma in Thailand: a population based survey in Rom Klao District, Bangkok. $\mathrm{Br}$ J Ophthalmol 2003; 87(9): 1069-1074.

4 Ramakrishnan R, Nirmalan PK, Krishnadas R, Thulasiraj $\mathrm{RD}$, Tielsch JM, Katz J et al. Glaucoma in a rural population of southern India: the Aravind comprehensive eye survey. Ophthalmology 2003; 110(8): 1484-1490.

5 Dandona L, Dandona R, Srinivas M, Mandal P, John RK, McCarty CA et al. Open-angle glaucoma in an urban population in southern India: the Andhra Pradesh eye disease study. Ophthalmology 2000; 107(9): 1702-1709.

6 Rotchford AP, Kirwan JF, Muller MA, Johnson GJ, Roux P. Temba glaucoma study: a population-based cross-sectional survey in urban South Africa. Ophthalmology 2003; 110(2): 376-382.

7 Rotchford AP, Johnson GJ. Glaucoma in Zulus: a population-based cross-sectional survey in a rural district in South Africa. Arch Ophthalmol 2002; 120(4): 471-478.

8 Grey RHB, Burns-Cox CJ, Hughes A. Blind and partial sight registration in Avon. Br J Ophthalmol 1989; 73: 88-94.

9 Thompson JR, Du L, Rosenthal AR. Recent trends in the registration of blindness and partial sight in Leicestershire. Br J Ophthalmol 1989; 73: 95-99.

10 Bamashmus MA, Matlhaga B, Dutton GN. Causes of blindness and visual impairment in the West of Scotland. Eye 2004; 18: 257-261.

11 King AJW, Reddy A, Thompson JR, Rosenthal AR. The rates of blindness and of partial sight registration in glaucoma patients. Eye 2000; 14: 613-619. 
12 Crabb DP, Viswanathan AC, McNaught AI, Poinoosawmy D, Fitzke FW, Hitchings RA et al. Simulating binocular visual field status in glaucoma. Br J Ophthalmol 1998; 82: 1236-1241.

13 Fuchs J, Nissen KR, Goldschmidt E. Glaucoma blindness in Denmark. Acta Ophthalmologica 1992; 70: 73-78.

14 Quigley HA, Vitale S. Models of open-angle glaucoma prevalence and incidence in the United States. Invest Ophthalmol Vis Sci 1997; 38: 83-91.
15 Quigley HA, Tielsch JM, Katz J, Sommer A. Rate of progression in open-angle glaucoma estimated from crosssectional prevalence of visual field damage. Am J Ophthalmol 1996; 122: 355-363.

16 Nelson-Quigg JM, Cello K, Johnson CA. Predicting binocular visual field sensitivity from monocular visual field results. Invest Ophthalmol Vis Sci 2000; 41(8): 2212-2221. 